\title{
Long-term Safety and Efficacy of Levodopa-Carbidopa Intestinal Gel in Advanced Parkinson's Disease
}

Hubert H. Fernandez, MD, ${ }^{1}$ James T. Boyd, MD, ${ }^{2}$ Victor S. C. Fung, MBBS, PhD ${ }^{3}$ Mark F. Lew, MD, ${ }^{4}$ Ramon L. Rodriguez, MD, ${ }^{5}$ John T. Slevin, MD, ${ }^{6}$ David G. Standaert, MD, PhD, ${ }^{7}$ Cindy Zadikoff, MD, ${ }^{8}$ Arvydas D. Vanagunas, MD,${ }^{8}$ Krai Chatamra, PhD, ${ }^{9}$ Susan Eaton, PharmD, ${ }^{9}$ Maurizio F. Facheris, MD, ${ }^{9}$ Coleen Hall, MS,${ }^{9}$ Weining Z. Robieson, $\mathrm{PhD},{ }^{9}$ Janet Benesh, BSMT, ${ }^{9}$ Alberto J. Espay, $\mathrm{MD}^{10}$

${ }^{1}$ Center for Neurological Restoration, Cleveland Clinic, Cleveland, $\mathrm{OH}$, United States;

${ }^{2}$ University of Vermont Larner College of Medicine, Burlington, VT, United States; ${ }^{3}$ Westmead Hospital and Sydney Medical School, Sydney, Australia; ${ }^{4}$ Keck/ University of Southern California School of Medicine, Los Angeles, CA, United States; ${ }^{5}$ University of Florida College of Medicine, Gainesville, FL, United States, and Orlando Veteran's Affairs Medical Center, Orlando, FL, United States; ${ }^{6}$ University of Kentucky Medical Center, Lexington, KY, United States; ${ }^{7}$ University of Alabama at Birmingham, Birmingham, AL, United States; ${ }^{8}$ Feinberg School of Medicine, Northwestern University, Chicago, IL, United States; ${ }^{9}$ AbbVie Inc., North Chicago, IL, United States; ${ }^{10}$ University of Cincinnati Academic Health Center, Cincinnati, $\mathrm{OH}$, United States

Correspondence to: Dr. Hubert H Fernandez, Center for Neurological Restoration, Cleveland Clinic, 9500 Euclid Avenue, S-3, Cleveland, OH 44195, USA; Phone: +1 216445 1108; Email: fernanh@ccf.org

Word Count: 3,700 words (maximum 3,700 words)

Key Words: Parkinson's disease, levodopa-carbidopa intestinal gel, percutaneous endoscopic gastrojejunostomy, safety, infusion, clinical trial

Running Title: Long-term safety and efficacy of LCIG 
Funding agencies: This study was funded by AbbVie Inc. AbbVie participated in the study design, research, data collection, analysis, and interpretation of data, writing, reviewing, and approving this manuscript for publication.

Financial Disclosures/Conflicts of Interest Related to Publication/AbbVie: H.H.F. has received research support from and has served as consultant/scientific advisor and lecturer for AbbVie. J.T.B. has received research support from and has served as a consultant/scientific advisor for AbbVie. V.S.C.F. has received research support from AbbVie. M.F.L. has received research support from and has served as a consultant, advisor, and/or lecturer for AbbVie. R.L.R. has received research support from AbbVie and honoraria from AbbVie for an educational symposium. J.T.S. has received research support from and has served as an advisor for AbbVie. D.G.S. is an investigator in studies funded by AbbVie and has served as a consultant or received honoraria from AbbVie; honoraria paid by AbbVie were for activities unrelated to authorship. C.Z. has received educational grants from and has served as an advisor for AbbVie. A.D.V. has received compensation from AbbVie for consultancy and advisory board services. C.H., W.Z.R., M.F.F., and J.B. are employees of AbbVie, and hold AbbVie stock and/or stock options. K.C. and S.E. are former employees of AbbVie. A.J.E. has received compensation as a consultant/scientific advisory board member from AbbVie. 


\section{ABSTRACT:}

Background: Levodopa-carbidopa intestinal gel (designated as carbidopa-levodopa enteral suspension in the United States) provides stable plasma levodopa concentrations and reduces motor fluctuations in advanced Parkinson's disease (PD) patients through continuous delivery of levodopa via percutaneous endoscopic gastrojejunostomy. We report long-term safety and efficacy outcomes from an open-label phase 3 treatment program.

Methods: PD patients ( $\mathrm{N}=262)$ who completed a 12-week double-blind study and its 52-week open-label extension, or a separate 54-week open-label study, were enrolled in this ongoing, phase 3, open-label, multinational study (NCT00660673). Safety and efficacy assessments were collected every 6 months.

Results: The mean total duration of exposure to levodopa-carbidopa intestinal gel was 4.1 years (range: 1.2 to 6.9 years). The overall discontinuation rate was $34 \%$ (average annual discontinuation rate: $10 \%)$. Although most patients (94\%) reported an adverse event, the rate of adverse events decreased over time; $53 \%$ experienced a serious adverse event. Of patients in this extension study, $54 \%$ required jejunal tube replacement during the study; $37 \%$ required percutaneous endoscopic gastrostomy tube replacement. Most patients were on levodopa monotherapy. Patients maintained reductions in "off" time and increases in mean "on" time without dyskinesia from initial LCIG infusion to study endpoint $(P<0.001 ; n=81)$. Activities of daily living and quality of life assessments demonstrated significant improvements that persisted through the study. 
Conclusions: This long-term study demonstrates sustained and clinically meaningful benefits from levodopa-carbidopa intestinal gel in advanced PD patients. Although adverse event rates decreased over time, vigilance is required for device-related complications and adverse events. 


\section{Introduction}

As Parkinson's disease (PD) progresses, nearly $90 \%$ of patients report disabling motor complications after 9 years of levodopa treatment, ${ }^{1}$ including fluctuations between adequate treatment ("on" time) and poor mobility ("off" time). ${ }^{2}$ These complications may be attributed to unstable levodopa plasma concentrations because of its short half-life, a narrowing therapeutic window, and erratic gastric emptying. ${ }^{2-4}$ To overcome irregular levodopa concentrations after oral administration, levodopa-carbidopa intestinal gel (LCIG, designated in the United States as carbidopa-levodopa enteral suspension) continuously delivers levodopa via percutaneous endoscopic gastrojejunostomy (PEG-J). LCIG is used in PD patients who experience motor fluctuations not adequately controlled by oral dopaminergic medications. Pharmacokinetic studies demonstrate more consistent plasma levodopa concentrations with direct intestinal levodopa administration compared with oral formulations. ${ }^{5,6}$

Previous long-term LCIG safety and efficacy studies were limited by retrospective (-9 $^{\text {or }}$ registry ${ }^{10,11}$ study design, and small data sets. ${ }^{12-14}$ Recently, larger randomized, controlled and open-label prospective studies demonstrated safety and efficacy of LCIG treatment through 1 year, ${ }^{15-17}$ and safety was demonstrated for an average of 2 years in an open-label extension study. ${ }^{18}$ LCIG treatment has consistently demonstrated significant reductions in "off" time and improvements in "on" time without increasing troublesome dyskinesia. ${ }^{15-17}$ We report our findings on patients previously enrolled in phase 3 LCIG studies ${ }^{15-17}$ who were allowed continued access to LCIG treatment through this open-label study until treatment became commercially available in each patient's home country. This is the longest prospective multinational study that presents LCIG safety and efficacy for advanced PD patients, with a mean follow-up of 4.1 years. 


\section{Methods}

\section{Study Design and Treatment}

Advanced PD patients with motor fluctuations not adequately controlled by oral dopaminergic medications, who completed either a 12-week double-blind study ${ }^{16}$ (NCT00660387/NCT0357994) and its 52-week open-label extension ${ }^{17}$ (NCT00360568), or a separate 54-week open-label study ${ }^{15}$ (NCT00335153), were eligible for enrollment in this ongoing, phase 3, open-label, multinational study (NCT00660673). Enrollment began in November 2009 and was completed in October 2012. Patients could remain in the study until they were transitioned to a locally available commercial product. Patients were enrolled from Australia, Canada, Czech Republic, Israel, New Zealand, Poland, Portugal, the Russian Federation, Thailand, the United Kingdom, and the United States. Patients were excluded if they had any medical, laboratory, psychiatric, or surgical issues deemed by the investigator to be clinically significant and which could interfere with the patient's participation in the study.

Patients received LCIG continuously administered through PEG-J tubing directly into the jejunum via a portable pump for 16 hours/day. Patients' initial LCIG dose was the same as that received at the end of their previous open-label LCIG study. Subsequent dose adjustments were made by the investigator as clinically indicated. In addition to a morning dose (to prime the intestinal tube and rapidly achieve the therapeutic dose) and the continuous infusion, patients could self-administer additional doses of LCIG to address immediate subjective needs (eg, motor function deterioration). Patients could use oral levodopa-carbidopa only for scheduled or supplemental bedtime/overnight doses after the pump was disconnected for the night. 
This study was conducted in accordance with the Good Clinical Practice Guideline as defined by the International Council on Harmonization, The Declaration of Helsinki, and all applicable federal and local regulations and institutional review boards.

\section{Assessments}

\section{Safety Assessment}

Patients had scheduled study visits every 6 months. Subjects transitioning to commercial product had a final study visit. Subjects discontinuing LCIG had their PEG-J removed and a final follow-up 1 week later. At each study visit, safety was assessed via physical examinations that included stoma site inspection, neurological examinations, vital sign and weight measurements, and excessive daytime sleepiness assessments. Laboratory tests to evaluate selective B vitamin deficiencies were added in Protocol Amendment 2 (July 2011); because some patients were already enrolled at that time, not all patients had vitamin levels assessed. Impulsive behavior was assessed using the Minnesota Impulsive Disorders Interview (MIDI). Annually, the PEG-J was evaluated by a gastroenterologist. An electrocardiogram and clinical laboratory tests were performed at the final study visit. Use of vitamin supplementation was made at the investigator's discretion, regardless of laboratory values. Adverse events (AEs) and product complaints could be reported at any time during the study.

\section{Efficacy Assessment}

Protocol amendment 4 (December 2013) for the extension study added the following efficacy assessments that had been used in the qualifying studies for patients at US sites: the PD symptom diary ${ }^{19,20}$ (PD diary), Unified Parkinson's Disease Rating Scale (UPDRS) and the Parkinson's Disease Questionnaire-39 (PDQ-39). 


\section{Statistical Analysis}

All patients who received at least one infusion of LCIG in the current study were included in the safety population. The subset of patients who were enrolled in the United States and had at least one efficacy assessment in the current study were included in the efficacy population. The last assessment in the previous LCIG study served as baseline for the current study. The change in efficacy measures from baseline in the current study as well as from the time of each patient's initial LCIG infusion in one of the previous studies was evaluated.

AEs were coded using the Medical Dictionary for Regulatory Activities (MedDRA, version 14.0). AEs could be coded to more than one preferred term. For example, AEs coded to "complication of device insertion" could also be coded to one or more term descriptive of the event (eg, “abdominal pain”, “duodenal ulcer”, “abdominal discomfort”, or "bezoar”). This manuscript includes only new AEs reported in this long-term, open-label study. Standardized search strategies based on MedDRA preferred terms identified AEs of special interest in the following categories: weight loss, polyneuropathy, and procedure/device-associated events. The polyneuropathy-related search included preferred terms in either the Peripheral Neuropathy or Guillain-Barre Syndrome standardized MedDRA query (narrow search), such as polyneuropathy, decreased vibratory sense, peripheral neuropathy, peripheral sensory neuropathy, neuralgia, demyelinating polyneuropathy, and sensory disturbance.

Efficacy variables based on the PD diary included "off" time, "on" time without troublesome dyskinesia, and "on" time with troublesome dyskinesia. "On" time without troublesome dyskinesia included "on" time without dyskinesia as well as "on" time with non-troublesome dyskinesia. PD diary times were normalized to a 16-hour waking day and averaged for the three 
days prior to each study visit. A one-sample paired $t$ test assessed significance in change of efficacy measures. Data are presented through the 30 September 2015 cutoff date. 


\section{Results}

\section{Patients}

A total of 262 patients from 11 countries participated in this open-label extension study and contributed data for the safety dataset (Fig. 1). Most patients were male (62\%), and $43 \%$ were from the United States (Table 1). Only patients from the United States $(n=86)$ were included in the efficacy subset. These patients had similar baseline characteristics to the overall safety population. As of the cutoff date, 110 patients (42\%) had completed the study and were transitioned to commercially available LCIG; 89 (34\%) had prematurely discontinued LCIG for any reason, including $A E(24 \%)$, lack of efficacy (3\%), withdrawn consent (6\%), administrative reasons $(<1 \%)$, and protocol violations $(<1 \%)$. As of the cut-off date, 63 patients $(24 \%)$ were still participating in the study because they lived in countries where LCIG was not yet commercially available.

The mean total exposure to LCIG in this extension study and previous study was 4.1 years (median: 4.3 years; range: 1.2 to 6.9 years). A total of 196 patients (75\%) were exposed for at least 2 years in this study (plus 1 year in the preceding studies); overall treatment with LCIG was 1081 patient-years with 800 patient-years in this extension study. The average overall discontinuation rate was $10 \% / y e a r$. For the efficacy population, the mean (standard deviation [SD]) total daily dose of levodopa was $1588(651) \mathrm{mg}$ at study baseline $(n=71)$ and $1783(768)$ mg at study endpoint. During the study, most patients (ranging from $86 \%$ in year 1 to $92 \%$ in year 5) were on levodopa-carbidopa monotherapy (LCIG and oral levodopa-carbidopa), with half (ranging from $47 \%$ to $53 \%$ ) not taking any other PD medications in addition to LCIG (Supplemental Table 1). At data cutoff, 30 of 71 patients used an extra LCIG dose (average of $141 \mathrm{mg} /$ day). 


\section{Safety}

\section{Adverse Events}

Most patients (94\%) had an AE during the study; the most frequently reported AEs are shown in Table 2. Events reported as "postoperative" as well as the term "complication of device insertion" were not always temporally linked to the procedure; "postoperative" was not defined with a specific length of time and referred to an event (eg, wound infection) that occurred any time after the procedure. The number of patients with one or more AEs declined over time (year 1: 79\%; year 2: 78\%; year 3: 69\%; year 4: 61\%; year 5: 48\%). Events associated with the procedure/device included postoperative wound infection, excessive granulation tissue, incision site erythema, complication of device insertion, procedural site reaction, abdominal pain, and procedural pain; the prevalence of these events decreased over time (Fig. 2a). Half (53\%) of the safety population experienced a serious $A E$; the most common serious $A E s$ are shown in Table 2. Patients experienced minimal changes in orthostatic blood pressure during the study (mean [SD] change from baseline: systolic, 0.2 [17.9] $\mathrm{mmHg}$; diastolic, -0.2 [13.4] $\mathrm{mmHg}$ ).

\section{Discontinuations Due to Adverse Events}

AEs led to discontinuations in 62 patients (24\%), including $38(15 \%)$ deaths. Of AEs leading to discontinuation, 14 patients $(5 \%)$ discontinued because of a procedure/device-related AE. The most common AEs leading to discontinuation were complication of device insertion $(n=5,2 \%)$, death of unknown causes $(n=5,2 \%)$, pneumonia $(n=4,2 \%)$, cardiac arrest $(n=3,1 \%)$, myocardial infarction $(n=3,1 \%)$, and reemergence of motor deficits often due to interruption in drug delivery $(n=3,1 \%)$. 


\section{Weight Loss}

The mean (SD) change from baseline in weight was a loss of $3.96(6.68) \mathrm{kg}$ (range: a loss of $30.7 \mathrm{~kg}$ to a gain of $12.3 \mathrm{~kg})$. Less than half $(48.4 \%)$ of patients experienced weight loss greater than $7 \%$. Weight loss was reported as an AE at the investigator's discretion. Weight lossrelated $A E$ terms including weight decreased, dysphagia, decreased appetite, hypophagia, and malnutrition were reported in 47 patients (18\%); 36 patients (14\%) reporting weight decreased. Weight loss was considered serious in 10 (3.8\%) patients (8 weight decreased and 1 each of dysphagia and malnutrition); only one case (dysphagia) led to discontinuation.

\section{Polyneuropathy}

Polyneuropathy-related symptoms were reported in 22 patients (8\%). One polyneuropathy event was considered serious. None of these polyneuropathy-related events led to study discontinuation. During the current study, polyneuropathy-related AE rates were relatively constant (year 1: 2.3\%; year 2: 3.0\%; year 3: 3.0\%; year 4: $1.9 \%$; year 5: $1.2 \%$ ).

Mean laboratory values for vitamin B6, vitamin B12, folic acid, and methylmalonic acid were in normal ranges for all patients with recorded laboratory values; mean homocysteine levels were higher than the normal range (Supplemental Table 2). Of the 22 patients who experienced polyneuropathy-related AEs, mean laboratory values were in normal ranges, except for homocysteine. The percentage of patients with polyneuropathy who had laboratory values above or below the normal ranges was like rates seen in the overall population. Nineteen of the 22 patients with polyneuropathy-related events were on vitamin supplementation. Of the 22 patients who experienced a polyneuropathy $A E$, only one patient experienced such an $A E$ in their previous LCIG study. This patient reported polyneuropathy and sensory disturbance on day 309 after LCIG initiation; vitamin levels were not collected at that time. 


\section{Impulsive and Compulsive Behaviors}

Impulse control behaviors reported based on the MIDI were ( $[\%]$ patients at study baseline and study endpoint, respectively): pathological gambling (1 [0.4\%] and 1 [0.4\%]), compulsive buying (1 [0.4\%] and 2 [0.8\%]), and compulsive sexual behavior (1 [0.4\%] and $13[5.1 \%])$.

\section{Device Complaints and Tube Replacements}

Device complaints (ie, related to pump and tube complaints, but not necessarily associated with an $A E$ ) occurred in 244 patients (93\%); the most common device complaints included device malfunction (85\%), device occlusion (57\%), and device dislocation (56\%). Of note, device malfunction could be double-coded to other complaints, including device occlusion and device dislocation. The number of patients with device complaints associated with AEs generally decreased over time (Fig. 2b). Of the 244 patients with device complaints, $80 \%$ had their pump replaced. The number of patients with one or more device complaint(s) generally decreased over time (Supplemental Fig. 1). Overall, 167 patients (63.7\%) had one or more device complaint(s) that resulted in either PEG or $\mathrm{J}$ tube replacement (average of $29.3 \%$ per year, range: $25.3 \%$ to $32.7 \%$ ).

More than half $(n=146,56 \%)$ of patients did not require PEG tube replacement; $86(33 \%)$ had one PEG tube replacement and $30(11 \%)$ had more than one PEG tube replacement (Supplemental Fig. 2a). Approximately one-third $(n=88,34 \%)$ of the patients did not require $J$ tube replacement, 69 (26\%) had one J tube replacement, and 105 (40\%) had more than one J tube replacement. The average (SD) PEG tube duration was 790.2 (438.1) days (range: 55 to 1941 days); the average (SD) J tube duration was 575.2 (399.1) days (range 55 to 1941 days). PEG and $\mathrm{J}$ tube replacements occurred at a relatively constant rate during this multiyear study 
(Supplemental Fig. 2b). The average annual rate of PEG and $\mathrm{J}$ tube replacements was $15.7 \%$ and $30.2 \%$, respectively.

\section{Deaths}

There were 38 deaths (15\%), of which two (intestinal dilatation and cardiac arrest) were considered by the investigator to be possibly related to treatment (described in detail previously ${ }^{18}$ ). The remaining 36 deaths were deemed unrelated to treatment and were mostly caused by complications of disease progression and advancing age.

\section{Efficacy}

Patients in the efficacy cohort (US patients only) experienced a significant decrease of nearly 4 hours in mean daily hours of "off" time from prior to initial LCIG infusion to study endpoint $(P<.001)$, with no significant change from study baseline to endpoint (Fig. 3a). Patients also experienced a significant increase of approximately 4 hours in mean daily hours of "on" time without troublesome dyskinesia $(P<.001$; Fig. 3b) that was maintained. Patients' mean daily "on" time with troublesome dyskinesia was significantly lower (decrease of 0.6 hours) from prior to initial LCIG infusion to study baseline $(P<.05$; Fig. 3c); however, this reduction was not maintained during the extension study.

UPDRS total score, as well as part II (activities of daily living) and part III (motor) scores, demonstrated significant improvements (mean score improvements of 3.03 and 4.77 , respectively) from prior to initial LCIG infusion to study baseline, that persisted through the remainder of the study (Supplemental Table 3). Similarly, PDQ-39 summary index scores 
demonstrated a mean improvement of 8.1 from prior to initial LCIG infusion to study baseline that persisted through study endpoint. 


\section{Discussion}

This study includes follow-up of patients who initiated LCIG treatment in a previous randomized controlled trial and an open-label trial, thus our findings are limited by differences in study designs and selection biases of the original studies from which patients were recruited. Patients were recruited from specialized movement disorders centers and therefore may be managed differently than patients in typical hospital settings. Efficacy was only assessed in a subset of the population in an open-label setting, which may overestimate the true benefit of LCIG beyond that reported in the parent studies. The study-required AE reporting may be artificially inflated; however, patient-monitoring in this study is closer to clinical practice than the preceding studies with visits every 6 months and limited study-specific assessments. Despite these limitations, this is the longest prospective study to date evaluating safety and efficacy of LCIG, with an average infusion of 4.1 years (median: 4.3 years; range: 1.2 to 6.9 years). Although a high incidence of AEs was noted, long-term use was associated with a relatively low ${ }^{21}$ annual discontinuation rate (average: 10\% per year) with overall discontinuation rates similar to other long-term follow-up studies $^{8-10,13,21-28}$ and low incidence of serious AEs.

The most frequently reported AEs were associated with PEG-J complications (mainly related to stoma site maintenance), comorbidities associated with advanced PD (eg, fall, fracture), aging (eg, fractures, osteoarthritis), or dopaminergic therapy (eg, dyskinesia, insomnia). The overall safety profile was like that reported in a recent integrated summary of safety of an average LCIG exposure of just over 2 years, ${ }^{18}$ as well as prospective open-label ${ }^{10,11,13,25-29}$ and retrospective observational studies. ${ }^{7-9,14,22,23}$ A total of $36(14 \%)$ patients reported AEs related to weight loss, as was also reported in the integrated safety study (59 patients, $14 \%),{ }^{18}$ and was similar to rates reported in a retrospective survey of over 900 patients, ${ }^{23}$ and a prospective 
open-label routine-care study of 59 patients. ${ }^{11}$ Although impulse control disorders are common in PD patients, and were an exclusion to enrollment, the treatment-emergent rate in this study was low, with an increase in compulsive sexual behavior from study baseline to the end of the study from $0.4 \%$ to $5.1 \%$. Increased rates of impulse control disorders from study baseline to endpoint is not uncommon-a study assessing long-term rotigotine treatment identified a frequency of impulse control disorders of $0.6 \%$ after 6 months that increased to $9 \%$ after 6.5 years. $^{30}$

Nonambulatory patients with enteral tubes for nutrition have experienced an average $\mathrm{J}$ tube longevity of 3 to 6 months ${ }^{31}$; PD patients treated with LCIG are mobile and issues related to enteral tube longevity-(eg, tube displacement), would be expected. However, the PEG and J tubes were durable during LCIG treatment, with an average PEG and J tube duration of 2.2 years and 1.6 years, respectively. The average tube longevity in this study is greater than the average longevity of 12 months reported in a retrospective survey ${ }^{23}$ and was like the 2.4-year average longevity reported in an observational study. ${ }^{24}$ Generally, PEG and J tubes are replaced when needed; however, it is unknown how many tubes were replaced preventively, as a cosmetic measure (eg, deterioration in the tube appearance), or whether the PEG and J components were replaced independently. In addition, J tubes require replacement when PEG tubes are replaced. PEG and J tubes were each replaced a median one time in the study; patients with an unusual number of tube replacements (ie, more than five) generally had unique circumstances. For example, one patient with $12 \mathrm{~J}$ tube replacements had dementia and frequently pulled the tube out during confusional states. Other studies have cited increased dementia (which results in accidental removal of tubes) as a reason for discontinuation, ${ }^{28}$ and is a point of consideration when deciding if a patient should continue LCIG treatment. Patients 
who had device complaints commonly had their pump replaced to correct situations such as error codes.

The polyneuropathy rate in this population $(3.4 \%)$ is similar to what has been reported in observational studies of patients treated with LCIG (rates of $3 \%$ reported in 2 studies, ${ }^{10,29}$ $5.4 \%,{ }^{27}$ and $7 \%{ }^{11}$ ); polyneuropathy rates were similar in the previous study (first year of treatment), with 18 patients (4.4\%) experiencing polyneuropathy. ${ }^{15}$ Similarly, 33 patients (8.0\%) experienced polyneuropathy-related events in the first year of treatment (previous study) ${ }^{15}$ and $22(8 \%)$ reported polyneuropathy-related symptoms in the current study, thus indicating a relatively constant rate of polyneuropathy and polyneuropathy-related events. These rates are much lower than the polyneuropathy rates of $14 \%,{ }^{13} 19 \%,{ }^{32}$ and $35 \%{ }^{25}$ reported in other prospective open-label studies. Despite reports that polyneuropathy with levodopa treatment may be associated with hyperhomocysteinemia and low levels of vitamin B6, vitamin B12, folic acid, and methylmalonic acid, ${ }^{33}$ patients experiencing polyneuropathy-related AEs in this study had mean laboratory values for these vitamins (except homocysteine) that were in normal ranges. However, most patients experiencing polyneuropathy-related AEs were on some level of vitamin supplementation. In addition, vitamin levels were not assessed after fasting. As a result, causality between LCIG, vitamin deficiencies, and polyneuropathy, cannot be established. Monitoring these vitamin levels was included as an amendment to the protocol for this study, thus baseline vitamin levels (before initial LCIG treatment in previous studies) were not available for most patients. Further systematic surveillance of vitamin levels is warranted to determine if vitamin supplementation may be considered in these patients.

Motor improvement from LCIG treatment was sustained and clinically meaningful as demonstrated by decreased "off" time, and increased "on" time without troublesome dyskinesia. 
Although these motor improvements were not supported by the UPDRS Part II scores and PDQ-39 summary index and subdomain scores, there was no motor worsening, as indicated by negligible change in the "on" state UPDRS Part III scores. Diminished improvement over time in PDQ-39 scores has been reported in long-term follow-up of patients treated with deep brain stimulation, which may reflect disease progression, treatment-related AEs (such as speech deterioration ${ }^{34}$ ), related comorbidities, or emergence of treatment-resistant symptoms. ${ }^{35}$ Because of general disease progression, advanced PD patients typically experience increases in UPDRS Part II scores; levodopa-treated patients have experienced PD progression in the form of an increase of 1.72 points/year in UPDRS Part ${ }^{136}$ (compared with an increase of 4.4 points/year in untreated patients ${ }^{37}$ ). In the current study, patients experienced an approximate rate of increase of 1.5 points/year. Further, these results are similar to other long-term openlabel studies, which reported slight increases in UPDRS Part II scores after 24 months of treatment, ${ }^{27}$ and no change in UPDRS Part II scores after 4 years. ${ }^{10} \mathrm{~A}$ retrospective study that compared outcomes in patients receiving LCIG, deep brain stimulation, or optimized standard oral therapy found that after an average of 5 years of treatment, the magnitude of worsening of UPDRS Part II scores was similar between patients receiving LCIG and deep brain stimulation and was lower for those patients receiving optimized oral therapy, indicating that LCIG and deep brain stimulation ameliorated the deterioration of activities of daily living that occurs in patients treated with optimized oral medication. ${ }^{14}$

In the initial 64 weeks of LCIG treatment in previous studies, the most common AEs were related to the procedure; ${ }^{15,16}$ however, with long-term use, patients experienced fewer of these complications. Most patients were on levodopa monotherapy, and their levodopa dose was constant or slightly increased over time, suggesting long-term tolerability of the dose needed to control motor symptoms/fluctuations. No correlation was noted between peripheral neuropathy 
and vitamin B levels, other than high homocysteine. Despite sustained motor improvements provided by LCIG in this advanced PD population, several comorbidities and complications, including death, do occur. Device-related complications were common and required vigilance, although they were generally reversible and seldom led to discontinuation. AEs were typical of the known risks of the PEG-J system, the advanced disease state, or the patient population. Therefore, despite the seemingly high rate of AEs, our long-term study suggested an overall favorable benefit/risk profile for long-term LCIG treatment. 


\section{Full Financial Disclosures for the Previous 12 Months}

This study was funded by AbbVie Inc. AbbVie participated in the study design, research, data collection, analysis, and interpretation of data, writing, reviewing, and approving the publication.

H.H.F. has no owner interest in any pharmaceutical company but has received research support from AbbVie, Acadia, Biotie/Acorda Therapeutics, Civitas, Kyowa/Prostrakan, the Michael J. Fox Foundation for Parkinson Research, Movement Disorders Society, NIH/NINDS, Parkinson Study Group, Rhythm, Synosia, and Teva. He has also received honoraria from Prime Education Inc, International Parkinson and Movement Disorders Society, Carling Communications, Medscape as a speaker in CME events. He has received honoraria from AbbVie, Biogen, GE Health Care, Inventiv, Kyowa Hakko Kirin, Lundbeck, Medscape, Merz Pharmaceuticals, Pfizer Pharmaceuticals, Sunovion, and Voyager, as a consultant. He has received a stipend from International Parkinson and Movement Disorders Society for serving as medical editor of the MDS Web Site; royalty payments from Demos Publishing for serving as a book author/editor; the Cleveland Clinic has contracts with Teva for his role as a co-principal investigator in SD-809 Tardive Dyskinesia global studies. He also serves as a member of the publication committee for Acorda Pharmaceuticals but does not receive any personal compensation for his involvement.

J.T.B. served as a consultant and/or scientific advisor for AbbVie, Auspex, Teva, Lundbeck, Neurocrine Biosciences, Chrono Therapeutics, and Medical Education Resources. He has received research support from the Michael J. Fox Foundation for Parkinson Research, NIH/NINDS, Auspex, Biotie, CHDI Foundation, Vaccinex, Teva, AbbVie, NeuroDerm, and Roche. 
V.S.C.F. receives a salary from NSW Health, has received research grants from the National Health and Medical Research Council of Australia and AbbVie, and is on Advisory Boards and/or has received travel grants from AbbVie, Allergan, Hospira, Ipsen, Lundbeck, Merz Pharmaceuticals, Mundi Pharma, and UCB.

M.F.L. has served as a consultant, advisor, and/or lecturer for Teva, US World Meds, Merz Pharmaceuticals, UCB, AbbVie, Acadia, Auspex, Lundbeck, Cynapsus, USWM, and Impax, and has received research support from NIH, AbbVie, Parkinson's Study Group, USWM, the Michael J. Fox Foundation for Parkinson Research, Synosia Pharmaceuticals, Merz Pharmaceuticals, Ipsen, Pharma Two B, Civitas, Biotie, and Cynapsus.

R.L.R. has no owner interest in any pharmaceutical company but has received research support from AbbVie, Allergan, Auspex, Biotie Therapeutics, Dystonia Coalition, Huntington Study Group, Ipsen, Merz Pharmaceuticals, National Parkinson Foundation, NIH/NINDS, Parkinson's Study Group, and Chelsea. He has also received honoraria from PeerView Institute for Medication Education, Merz Pharmaceuticals, Lundbeck, Chelsea, and the CME Meeting, and honoraria from AbbVie for an educational symposium.

J.T.S. has served as an advisor for AbbVie and Cynapsus Therapeutics Inc. He has also served as a speaker for Teva and AbbVie, and has received research support from AbbVie, Biotie, NIH, Parkinson Study Group, and the Veterans Administration.

D.G.S. is a member of the faculty of the University of Alabama at Birmingham and is supported by endowment and University funds. Dr. Standaert is an investigator in studies funded by Abbvie, Inc., the American Parkinson Disease Association, the Michael J. Fox Foundation for 
Parkinson Research, Alabama Department of Commerce, and NIH grants P01NS087997, P20NS087997, R25NS079188, P2CHD086851, and P30NS047466. He has a clinical practice and is compensated for these activities through the University of Alabama Health Services Foundation. In addition, since January 1, 2016 he has served as a consultant for or received honoraria from, Serina Therapeutics, Abbvie (honoraria paid by AbbVie were for activities unrelated to authorship), Voyager Therapeutics, the Michael J. Fox Foundation for Parkinson Research, The International Parkinson Disease and Movement Disorder Society, the National Institutes of Health, The American Institute for Biological Sciences, Rush University, Huntsville Hospital, UCSD, Voyager Therapeutics, and he has received royalties for publications from McGraw Hill, Inc.

C.Z. has served as an advisor for AbbVie, Teva, Cynapsus, Lundbeck, UCG, and Merz Pharmaceuticals, and has received honoraria from Teva, UCB, US World Meds, Cynapsus, AbbVie, Acadia, Lundbeck, and Merz Pharmaceuticals; honoraria paid by AbbVie were for activities unrelated to authorship. She has received educational grants from AbbVie, Teva, and Allergan.

A.D.V. has received compensation from AbbVie for consultancy and advisory board services. He has also received compensation for consultancy services from CVS/Caremark Pharmacy and Therapeutics Committee.

C.H., W.Z.R., M.F.F., and J.B. are employees of AbbVie, and hold AbbVie stock and/or stock options.

K.C. is a former employee of AbbVie and currently is employed by Lundbeck. 
S.E. is a former employee of AbbVie and currently is employed by ZS Pharma.

A.J.E. is supported by the NIH (1K23MH092735); he has received grant support from CleveMed/Great Lakes Neurotechnologies, and the Michael J Fox Foundation for Parkinson Research. He has also received personal compensation as a consultant/scientific advisory board member for AbbVie, Teva, Impax, Merz Pharmaceuticals, Pfizer, Acadia, Cynapsus, Solstice Neurosciences, Lundbeck, and US Worlds Meds. He has received royalties from Lippincott Williams \& Wilkins, Cambridge University Press, and Springer, and honoraria from UCB, US Worlds Meds, Lundbeck, Acadia, the American Academy of Neurology, Movement Disorders Society, and AbbVie for educational symposia. He also serves as associate editor of the Journal of Clinical Movement Disorders and is on the editorial board of Parkinsonism and Related Disorders. 


\section{Author Roles}

Specific roles in the project and manuscript preparation shown below are defined as follows:

1. Research project: A. Conception, B. Organization, C. Execution

2. Statistical analysis: A. Design, B. Execution, C. Review and Critique

3. Manuscript preparation: A. Writing of the first draft, B. Review and critique

H.H.F.: 1A, 1B, 1C, 2C, 3A, 3B

J.T.B.: 1B, 1C, 2C, 3A, 3B

V.S.C.F.: 1B, 1C, 2C, 3A, 3B

M.F.L.: 1B, 1C, 2C, 3A, 3B

R.L.R.: 1B, 1C, 2C, 3A, 3B

J.T.S.: 1B, 1C, 2C, 3A, 3B

D.G.S.: 1B, 1C, 2C, 3A, 3B

C.Z.: 1B, 1C, 2C, 3A, 3B

A.D.V.: 1B, 1C, 2C, 3A, 3B

K.C.: 1A, 2C, 3A, 3B

S.E.: 1B, 1C, 2C, 3A, 3B

M.F.F.: 1B, 1C, 2C, 3A, 3B

C.H.: 2A, 2B, 2C, 3A, 3B

W.Z.R.: 1A, 1B, 2A, 2B, 2C, 3A, 3B

J.B.: 1A, 1B, 2C, 3A, 3B

A.J.E.: 1B, 1C, 2C, 3A, 3B

Acknowledgments: Medical writing assistance was provided by Kelly M Cameron, PhD, of The JB Ashtin Group, Inc., who, on behalf of AbbVie Inc, provided assistance in writing the first draft and implemented author revisions throughout the editorial process. 


\section{References}

1. Ahlskog JE, Muenter MD. Frequency of levodopa-related dyskinesias and motor fluctuations as estimated from the cumulative literature. Mov Disord 2001;16(3):448-458.

2. Antonini A, Chaudhuri KR, Martinez-Martin P, Odin P. Oral and infusion levodopa-based strategies for managing motor complications in patients with Parkinson's disease. CNS Drugs 2010;24(2):119-129.

3. Contin M, Martinelli P. Pharmacokinetics of levodopa. J Neurol 2010;257(Suppl 2):S253261.

4. Lundqvist C. Continuous levodopa for advanced Parkinson's disease. Neuropsychiatr Dis Treat 2007;3(3):335-348.

5. Othman AA, Chatamra K, Mohamed ME, et al. Jejunal Infusion of levodopa-carbidopa intestinal gel versus oral administration of levodopa-carbidopa tablets in japanese subjects with advanced Parkinson's disease: pharmacokinetics and pilot efficacy and safety. Clin Pharmacokinet 2015;54(9):975-984.

6. Othman AA, Rosebraugh M, Chatamra K, Locke C, Dutta S. Levodopa-Carbidopa Intestinal Gel Pharmacokinetics: Lower Variability than Oral Levodopa-Carbidopa. J Parkinsons Dis 2017.

7. Bajenaru O, Ene A, Popescu BO, et al. The effect of levodopa-carbidopa intestinal gel infusion long-term therapy on motor complications in advanced Parkinson's disease: a multicenter Romanian experience. J Neural Transm (Vienna) 2016;123(4):407-414.

8. Nyholm D, Klangemo K, Johansson A. Levodopa/carbidopa intestinal gel infusion longterm therapy in advanced Parkinson's disease. Eur J Neurol 2012;19(8):1079-1085.

9. Valldeoriola F, Grandas F, Santos-Garcia D, et al. Long-term effectiveness of levodopacarbidopa intestinal gel in 177 Spanish patients with advanced Parkinson's disease. Neurodegener Dis Manag 2016;6(4):289-298.

10. Buongiorno M, Antonelli F, Camara A, et al. Long-term response to continuous duodenal infusion of levodopa/carbidopa gel in patients with advanced Parkinson disease: The Barcelona registry. Parkinsonism Relat Disord 2015;21(8):871-876. 
11. Zibetti M, Merola A, Artusi CA, et al. Levodopa/carbidopa intestinal gel infusion in advanced Parkinson's disease: a 7-year experience. Eur J Neurol 2014;21(2):312-318.

12. Bohlega S, Abou Al-Shaar H, Alkhairallah T, Al-Ajlan F, Hasan N, Alkahtani K. LevodopaCarbidopa Intestinal Gel Infusion Therapy in Advanced Parkinson's Disease: Single Middle Eastern Center Experience. Eur Neurol 2015;74(5-6):227-236.

13. Caceres-Redondo MT, Carrillo F, Lama MJ, et al. Long-term levodopa/carbidopa intestinal gel in advanced Parkinson's disease. J Neurol 2014;261(3):561-569.

14. Merola A, Romagnolo A, Zibetti M, Bernardini A, Cocito D, Lopiano L. Peripheral neuropathy associated with levodopa-carbidopa intestinal infusion: a long-term prospective assessment. Eur J Neurol 2016;23(3):501-509.

15. Fernandez $\mathrm{HH}$, Standaert DG, Hauser RA, et al. Levodopa-carbidopa intestinal gel in advanced Parkinson's disease: final 12-month, open-label results. Mov Disord 2015;30(4):500-509.

16. Olanow CW, Kieburtz K, Odin P, et al. Continuous intrajejunal infusion of levodopacarbidopa intestinal gel for patients with advanced Parkinson's disease: a randomised, controlled, double-blind, double-dummy study. Lancet Neurol 2014;13(2):141-149.

17. Slevin JT, Fernandez HH, Zadikoff $\mathrm{C}$, et al. Long-term safety and maintenance of efficacy of levodopa-carbidopa intestinal gel: an open-label extension of the double-blind pivotal study in advanced Parkinson's disease patients. J Parkinsons Dis 2015;5(1):165-174.

18. Lang AE, Rodriguez RL, Boyd JT, et al. Integrated safety of levodopa-carbidopa intestinal gel from prospective clinical trials. Mov Disord 2016;31(4):538-546.

19. Hauser RA, Deckers F, Lehert P. Parkinson's disease home diary: further validation and implications for clinical trials. Mov Disord 2004;19(12):1409-1413.

20. Hauser RA, Friedlander J, Zesiewicz TA, et al. A home diary to assess functional status in patients with Parkinson's disease with motor fluctuations and dyskinesia. Clin Neuropharmacol 2000;23(2):75-81.

21. Borgemeester RW, Drent M, van Laar T. Motor and non-motor outcomes of continuous apomorphine infusion in 125 Parkinson's disease patients. Parkinsonism Relat Disord 2016;23:17-22. 
22. Nyholm D, Lewander T, Johansson A, Lewitt PA, Lundqvist C, Aquilonius SM. Enteral levodopa/carbidopa infusion in advanced Parkinson disease: long-term exposure. Clin Neuropharmacol 2008;31(2):63-73.

23. Sensi M, Cossu G, Mancini F, et al. Which patients discontinue? Issues on Levodopa/carbidopa intestinal gel treatment: Italian multicentre survey of 905 patients with long-term follow-up. Parkinsonism Relat Disord 2017;38:90-92.

24. Calandrella D, Romito LM, Elia AE, et al. Causes of withdrawal of duodenal levodopa infusion in advanced Parkinson disease. Neurology 2015;84(16):1669-1672.

25. De Fabregues O, Dot J, Abu-Suboh M, et al. Long-term safety and effectiveness of levodopa-carbidopa intestinal gel infusion. Brain Behav 2017;7(8):e00758.

26. Kruger R, Lingor P, Doskas T, et al. An Observational Study of the Effect of LevodopaCarbidopa Intestinal Gel on Activities of Daily Living and Quality of Life in Advanced Parkinson's Disease Patients. Adv Ther 2017;34(7):1741-1752.

27. Palhagen SE, Sydow O, Johansson A, et al. Levodopa-carbidopa intestinal gel (LCIG) treatment in routine care of patients with advanced Parkinson's disease: An open-label prospective observational study of effectiveness, tolerability and healthcare costs. Parkinsonism Relat Disord 2016;29:17-23.

28. Udd M, Lyytinen J, Eerola-Rautio J, et al. Problems related to levodopa-carbidopa intestinal gel treatment in advanced Parkinson's disease. Brain Behav 2017;7(7):e00737.

29. Antonini A, Yegin A, Preda C, et al. Global long-term study on motor and non-motor symptoms and safety of levodopa-carbidopa intestinal gel in routine care of advanced Parkinson's disease patients; 12-month interim outcomes. Parkinsonism Relat Disord 2015;21(3):231-235.

30. Antonini A, Chaudhuri KR, Boroojerdi B, et al. Impulse control disorder related behaviours during long-term rotigotine treatment: a post hoc analysis. Eur J Neurol 2016;23(10):15561565.

31. Epstein M, Johnson DA, Hawes R, et al. Long-Term PEG-J Tube Safety in Patients With Advanced Parkinson's Disease. Clin Transl Gastroenterol 2016;7:e159.

32. Rispoli V, Simioni V, Capone JG, et al. Peripheral neuropathy in 30 duodopa patients with vitamins B supplementation. Acta Neurol Scand 2017. 
33. Muller T, van Laar T, Cornblath DR, et al. Peripheral neuropathy in Parkinson's disease: levodopa exposure and implications for duodenal delivery. Parkinsonism Relat Disord 2013;19(5):501-507 ; discussion 501.

34. Skodda S. Effect of deep brain stimulation on speech performance in Parkinson's disease. Parkinsons Dis 2012;2012:850596.

35. Weaver FM, Follett KA, Stern M, et al. Randomized trial of deep brain stimulation for Parkinson disease: thirty-six-month outcomes. Neurology 2012;79(1):55-65.

36. Evans JR, Mason SL, Williams-Gray $\mathrm{CH}$, et al. The natural history of treated Parkinson's disease in an incident, community based cohort. J Neurol Neurosurg Psychiatry 2011;82(10):1112-1118.

37. Parkinson Study G. Effects of tocopherol and deprenyl on the progression of disability in early Parkinson's disease. N Engl J Med 1993;328(3):176-183. 
TABLE 1. Demographics and baseline disease characteristics

\begin{tabular}{|c|c|c|}
\hline Characteristic, n (\%) & $\begin{array}{l}\text { Safety population } \\
\qquad N=262\end{array}$ & $\begin{array}{l}\text { Efficacy population }{ }^{a} \\
\qquad n=86\end{array}$ \\
\hline Age (years) mean (SD) & $64.1(8.9)$ & $65.1(8.3)$ \\
\hline Median (range) & $64(39-84)$ & $65(41-82)$ \\
\hline \multicolumn{3}{|l|}{ Sex, n (\%) } \\
\hline Male & $162(62)$ & $60(70)$ \\
\hline Female & $100(38)$ & $26(30)$ \\
\hline \multicolumn{3}{|l|}{ Country, n (\%) } \\
\hline United States & $113(43)$ & $86(100)$ \\
\hline Russian Federation & $43(16)$ & \\
\hline Czech Republic & $22(8)$ & \\
\hline Portugal & $14(5)$ & \\
\hline Thailand & $13(5)$ & \\
\hline Australia & $12(5)$ & \\
\hline Canada & $11(4)$ & \\
\hline United Kingdom & $11(4)$ & \\
\hline New Zealand & $10(4)$ & \\
\hline Israel & $9(3)$ & \\
\hline Poland & $4(2)$ & \\
\hline Mean (SD) weight, kg & $69.8(15.3)$ & $74.2(15.2)$ \\
\hline Median (range) & $68.3(40.0-121.4)$ & $70.68(47.3-121.4)$ \\
\hline Mean (SD) BMI, kg/m² & $24.2(4.2)^{\mathrm{b}}$ & $25.0(4.7)$ \\
\hline Median (range) & $23.6(16.4-37.3)^{b}$ & $23.9(17.6-37.3)$ \\
\hline Mean (SD) orthostatic systolic blood pressure ${ }^{c}, \mathrm{mmHg}$ & $-7.1(13.8)$ & $-9.9(14.2)$ \\
\hline Median (range) & $-5.0(-59.0-25.0)$ & $-8.0(-56.0-17.0)$ \\
\hline Mean (SD) orthostatic diastolic blood pressure ${ }^{\mathrm{c}}, \mathrm{mmHg}$ & $-1.7(10.1)$ & $-1.9(10.3)$ \\
\hline Median (range) & $-0.5(-66.0-41.0)$ & $0(-46.0-20.0)$ \\
\hline Mean (SD) duration of Parkinson's disease, years & $11.4(5.4)$ & $10.5(4.5)$ \\
\hline Median (range) & $10.4(1.5-31.3)$ & $9.6(1.7-24.9)$ \\
\hline
\end{tabular}

$\mathrm{SD}$, standard deviation; BMI, body mass index.

aPer protocol amendment, patients in the efficacy population were only from the United States.

${ }^{b} \mathrm{n}=259$.

${ }^{\mathrm{c} C h a n g e}$ from sitting to standing position. 
TABLE 2. Adverse events (AEs) and serious AEs

Parameter $^{\mathrm{a}, \mathrm{b}}$

$\mathrm{N}=262$

$\mathrm{n}(\%)$

AEs Occurring in $\geq 10 \%$ of Patients

Postoperative wound infection

$59(23)$

Vitamin B6 decreased

$58(22)$

Fall

$55(21)$

Urinary tract infection

$50(19)$

Blood homocysteine increased

$48(18)$

Excessive granulation tissue

$41(16)$

Incision site erythema

$38(15)$

Weight decreased

$36(14)$

Complication of device insertion ${ }^{c}$

$33(13)$

Parkinson's disease ${ }^{d}$

$33(13)$

Procedural site reaction

33 (13)

Nausea

$32(12)$

Depression

$30(11)$

Constipation

$29(11)$

Insomnia

$29(11)$

Abdominal pain

27 (10)

Dyskinesia

$27(10)$

Procedural pain

27 (10)

Serious AEs Occurring in $\geq 3 \%$ of Patients

Pneumonia

$17(6)$

Complication of device insertion ${ }^{c}$

Fall

$12(5)$ 
Pneumonia aspiration

Postoperative wound infection

Weight decreased

${ }^{\text {aA } A}$ single event could be coded to $\geq 1$ MedDRA preferred term descriptive of the event.

${ }^{b}$ Events associated with the procedure or device are italicized.

"Events with this term were most often additionally coded to "abdominal pain", "duodenal ulcer", "abdominal discomfort", or "bezoar".

${ }^{\mathrm{d} R e f e r s ~ t o ~ t h e ~ r e e m e r g e n c e ~ o f ~ P a r k i n s o n ' s ~ s y m p t o m s, ~ o f t e n ~ d u e ~ t o ~ a ~ p r o b l e m ~ w i t h ~ d r u g ~ d e l i v e r y . ~}$ 


\section{FIGURE LEGENDS}

FIG. 1. Disposition of patients. IR-LC, immediate-release oral levodopa-carbidopa; LCIG, levodopa-carbidopa intestinal gel.

FIG. 2. (a) Prevalence of AEs related to the procedure or device are reported for those AEs occurring in $\geq 5 \%$ of patients in any year of the study and (b) patients with $\geq 1$ device complaint associated with an $\mathrm{AE}$ over 5 years. $\mathrm{AE}$, adverse event.

FIG. 3. Mean daily hours of (a) "off" time, (b) "on" time without troublesome dyskinesia, and (c) "on" time with troublesome dyskinesia in US patients $(n=81)$ from the first LCIG study dose through the continued-access-to-treatment study. "Prior to initial LCIG infusion" occurred in a previous study, before the first LCIG infusion, and a portion of these patients $(n=19)$ were administered immediate-release levodopa-carbidopa for 12 weeks during the double-blind study prior to their first LCIG infusion in the following 52-week open-label extension study. "Study Baseline" refers to the last assessment in a previous study, which served as a baseline for this open-label extension study; "Endpoint" refers to measurements at the cutoff date for this openlabel, extension study. Error bars indicate standard deviation. ${ }^{* *} P<0.001 ;{ }^{*} P<0.05$.

LCIG, levodopa-carbidopa intestinal gel; TSD, troublesome dyskinesia. 
FIG. 1

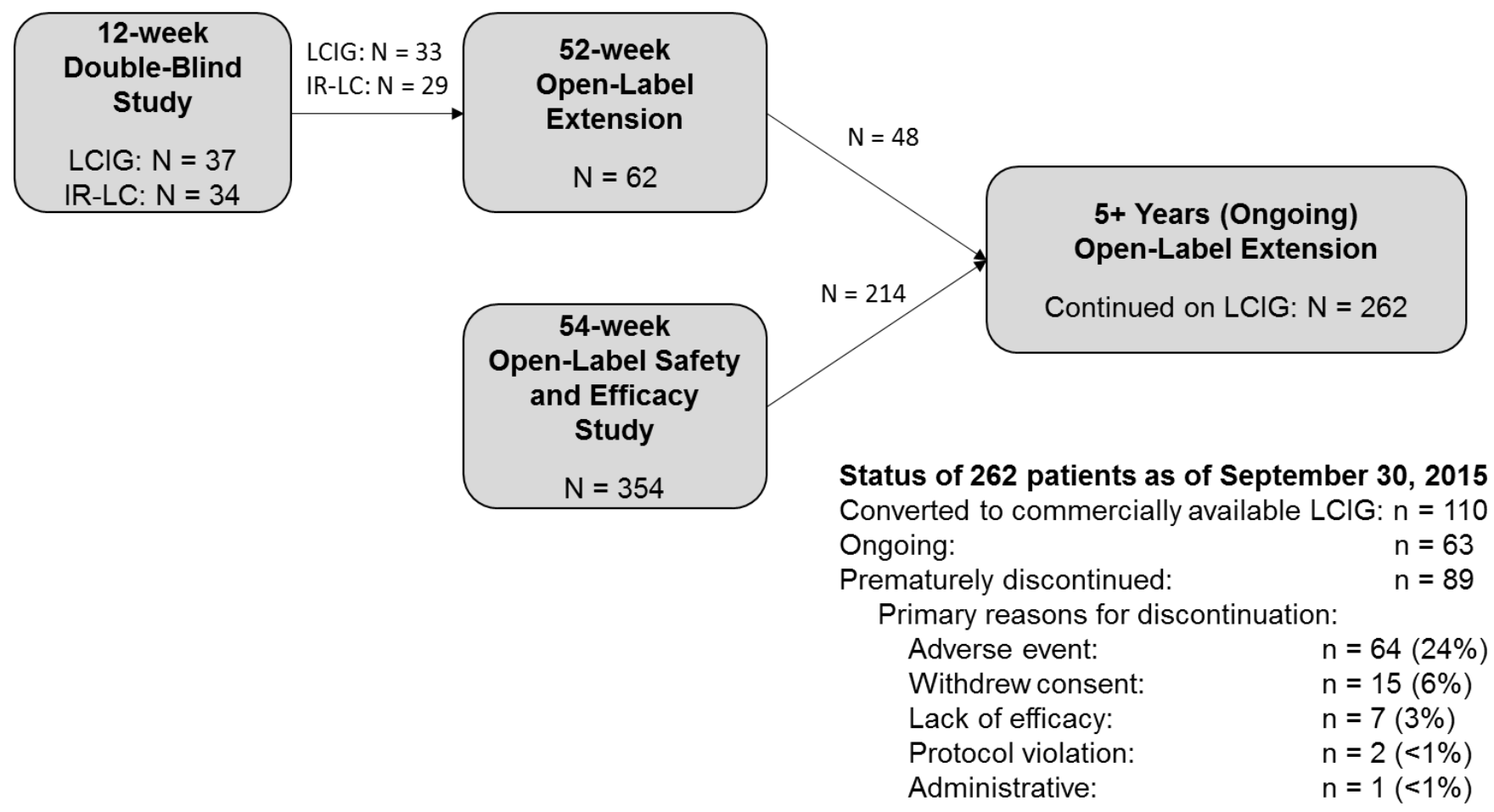


FIG. 2

a

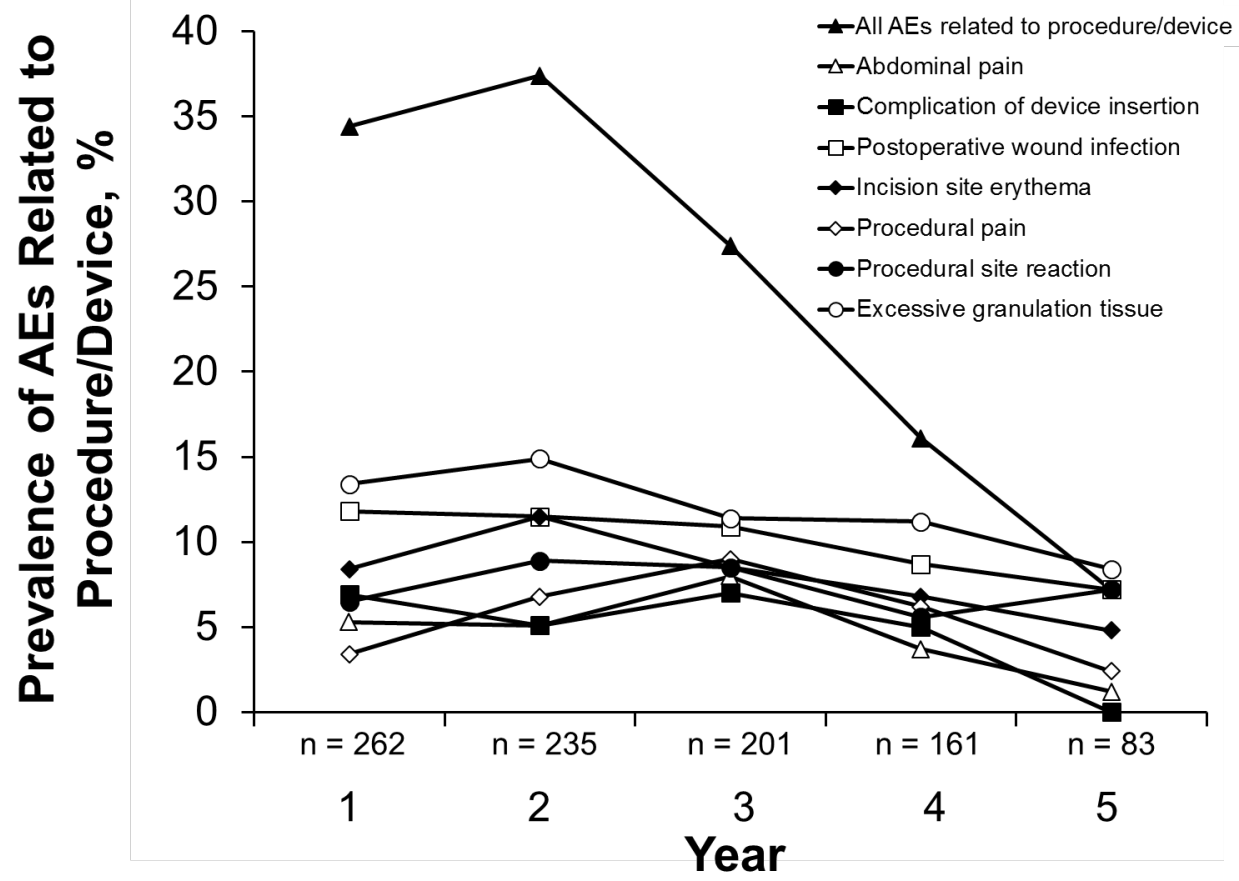

b

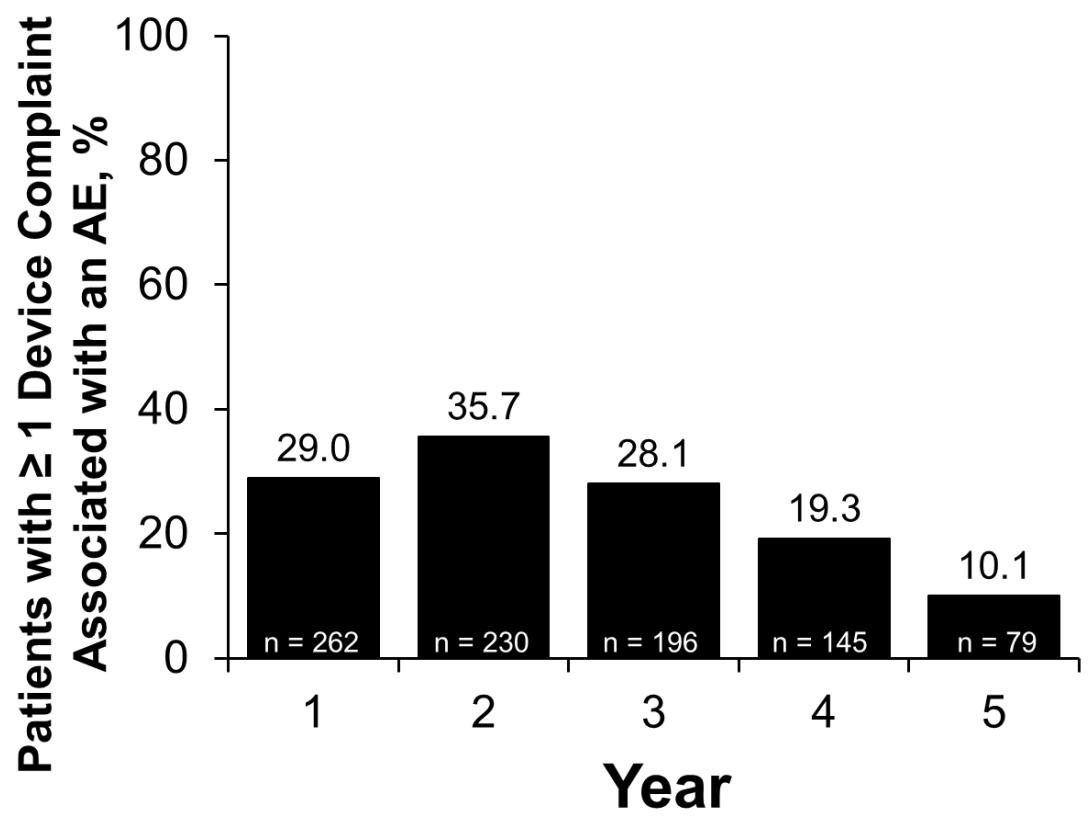


FIG. 3

a

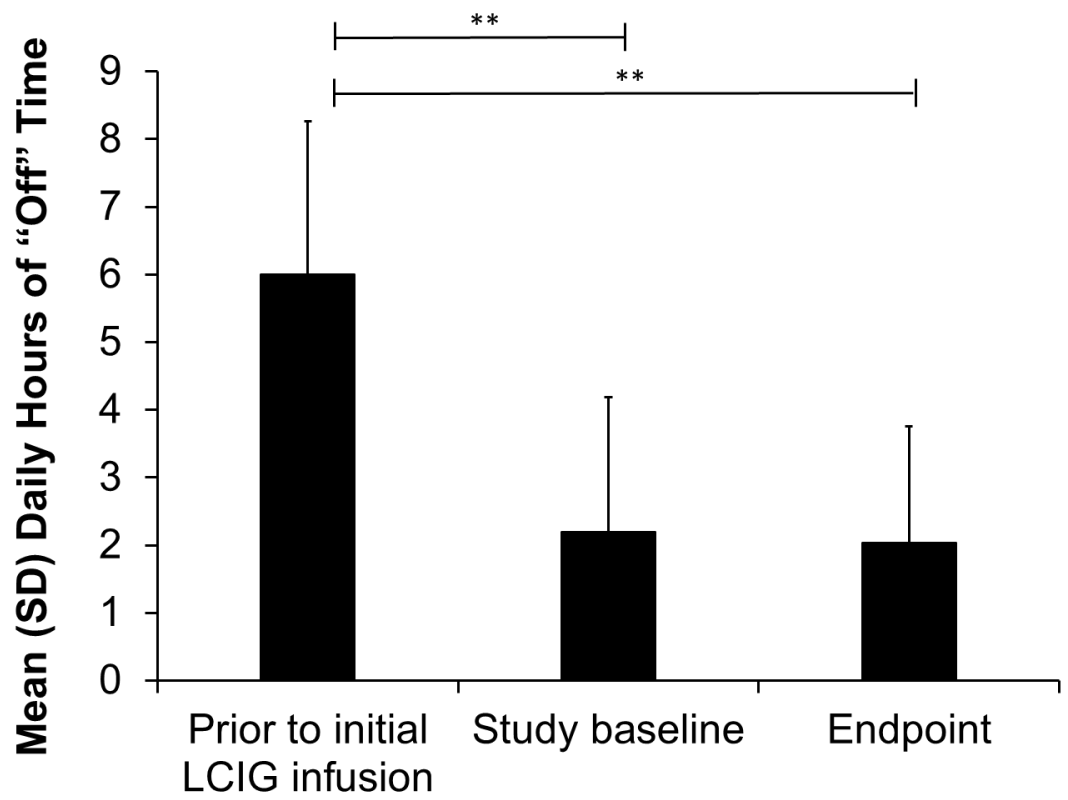

b

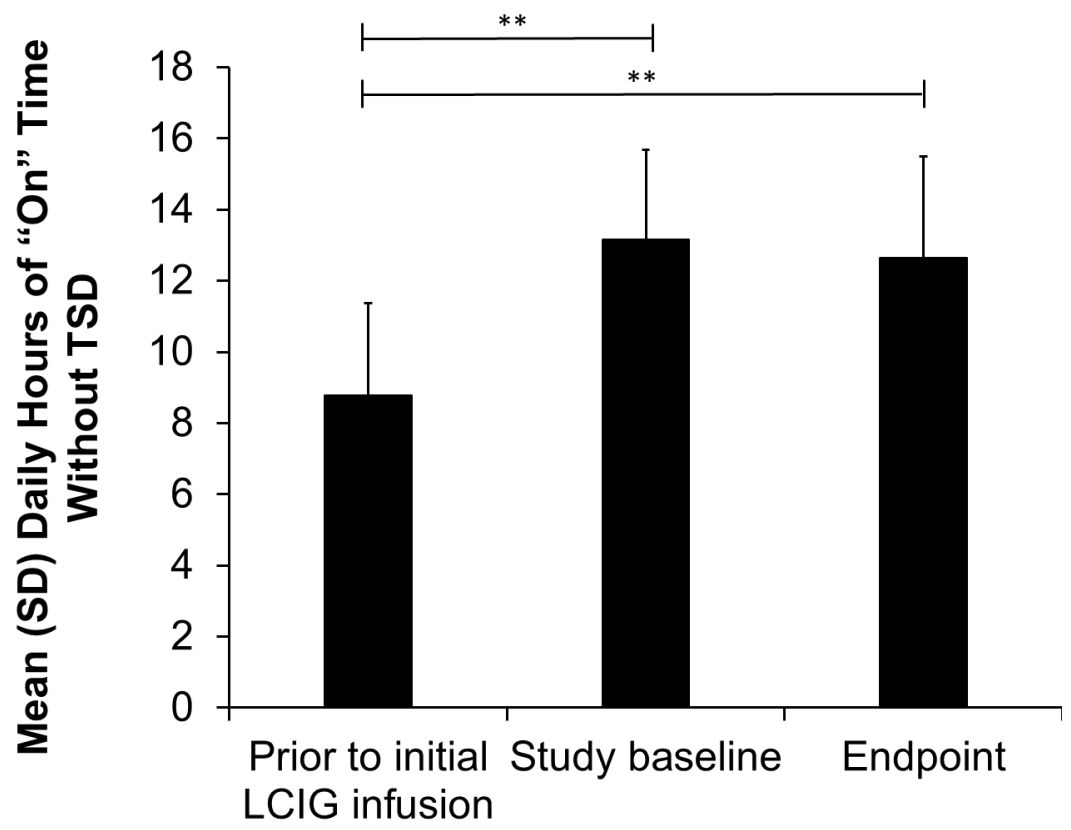




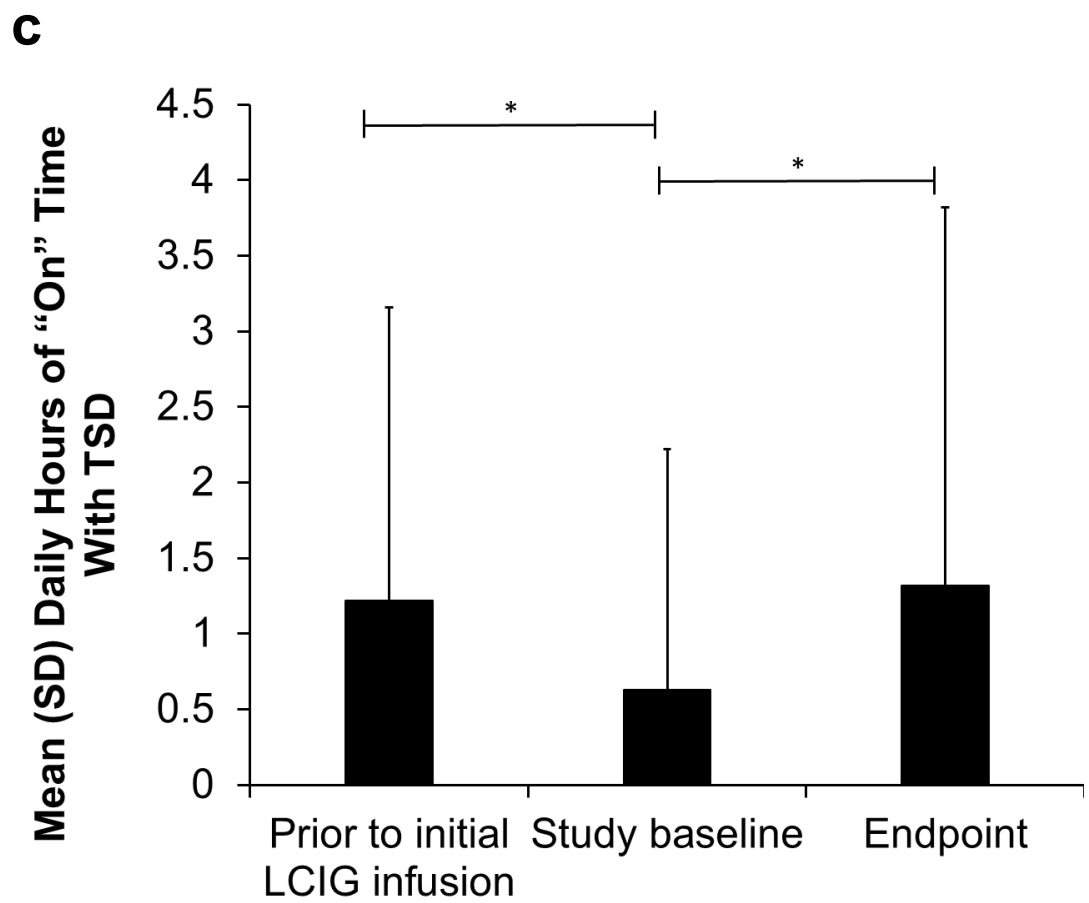




\section{SUPPLEMENTAL MATERIAL}

SUPPLEMENTAL TABLE 1. Concomitant anti-PD medications by year of treatment

\begin{tabular}{lcccccc} 
Treatment & \multicolumn{7}{c}{ Patients, $\mathrm{n}(\%)$} \\
$\mathrm{n}(\%)$ & $\begin{array}{c}\text { Day 1 } \\
(\mathrm{n}=262)\end{array}$ & $\begin{array}{c}\text { Year 1 } \\
(\mathrm{n}=262)\end{array}$ & $\begin{array}{c}\text { Year 2 } \\
(\mathrm{n}=230)\end{array}$ & $\begin{array}{c}\text { Year 3 } \\
(\mathrm{n}=196)\end{array}$ & $\begin{array}{c}\text { Year 4 } \\
(\mathrm{n}=145)\end{array}$ & $\begin{array}{c}\text { Year 5 } \\
(\mathrm{n}=79)\end{array}$ \\
$\begin{array}{l}\text { LCIG and oral } \\
\text { levodopa/carbidopa }\end{array}$ & $212(81)$ & $226(86)$ & $198(86)$ & $170(87)$ & $129(89)$ & $73(92)$ \\
LCIG only & $126(48)$ & $126(48)$ & $107(47)$ & $96(49)$ & $74(51)$ & $42(53)$ \\
Concomitant PD medications & $50(19)$ & $36(14)$ & $32(14)$ & $26(13)$ & $16(11)$ & $6(8)$
\end{tabular}

PD, Parkinson's disease; LCIG, levodopa-carbidopa intestinal gel.

ancluding patients who were on LCIG only without concomitant anti-parkinsonian medications and patients who only took oral levodopa-carbidopa as concomitant anti-parkinsonian medication. 
SUPPLEMENTAL TABLE 2. Laboratory values monitoring vitamin status

Patients with values outside normal range, $\mathrm{n}$

Mean (SD)

$(\%)$

\begin{tabular}{|c|c|c|c|c|c|c|c|}
\hline Parameter & $\begin{array}{c}\text { Central } \\
\text { laboratory } \\
\text { normal range }\end{array}$ & $\mathrm{n}$ & Study baseline ${ }^{a}$ & Endpoint & Change from baseline & $<\mathrm{LLN}^{\mathrm{b}}$ & $>U_{L N}{ }^{b}$ \\
\hline Vitamin B6, nmol/L & $20.0-125.0$ & 119 & $70.2(98.5)$ & $94.6(119.3)$ & $+24.4(128.7)$ & $151(72)$ & $98(46)$ \\
\hline Vitamin B12, pmol/L & $148.0-775.0$ & 126 & $389.2(435.4)$ & $562.8(975.8)$ & +173.5 (900.7) & $19(9)$ & $44(21)$ \\
\hline $\begin{array}{l}\text { Homocysteine, } \\
\mu \mathrm{mol} / \mathrm{L}\end{array}$ & $3.7-13.9$ & 126 & $19.5(9.4)$ & $23.6(12.8)$ & $+4.1(11.1)$ & $1(0.5)$ & $197(92)$ \\
\hline $\begin{array}{l}\text { Methylmalonic acid, } \\
\mu \mathrm{mol} / \mathrm{L}\end{array}$ & $0.0-0.4$ & 127 & $0.267(0.222)$ & $0.285(0.317)$ & $+0.018(0.223)$ & $\mathrm{N} / \mathrm{A}$ & $58(27)$ \\
\hline \multicolumn{8}{|c|}{ Patients experiencing polyneuropathy-related adverse events ${ }^{c}$} \\
\hline $\begin{array}{l}\text { Homocysteine, } \\
\mu \mathrm{mol} / \mathrm{L}\end{array}$ & $3.7-13.9$ & 13 & $19.14(10.52)$ & $24.68(13.25)$ & $+5.55(5.99)$ & 0 & $18(90)$ \\
\hline $\begin{array}{l}\text { Methylmalonic acid, } \\
\mu \mathrm{mol} / \mathrm{L}\end{array}$ & $0.0-0.4$ & 14 & $0.401(0.497)$ & $0.371(0.629)$ & $-0.030(0.233)$ & N/A & $5(25)$ \\
\hline
\end{tabular}




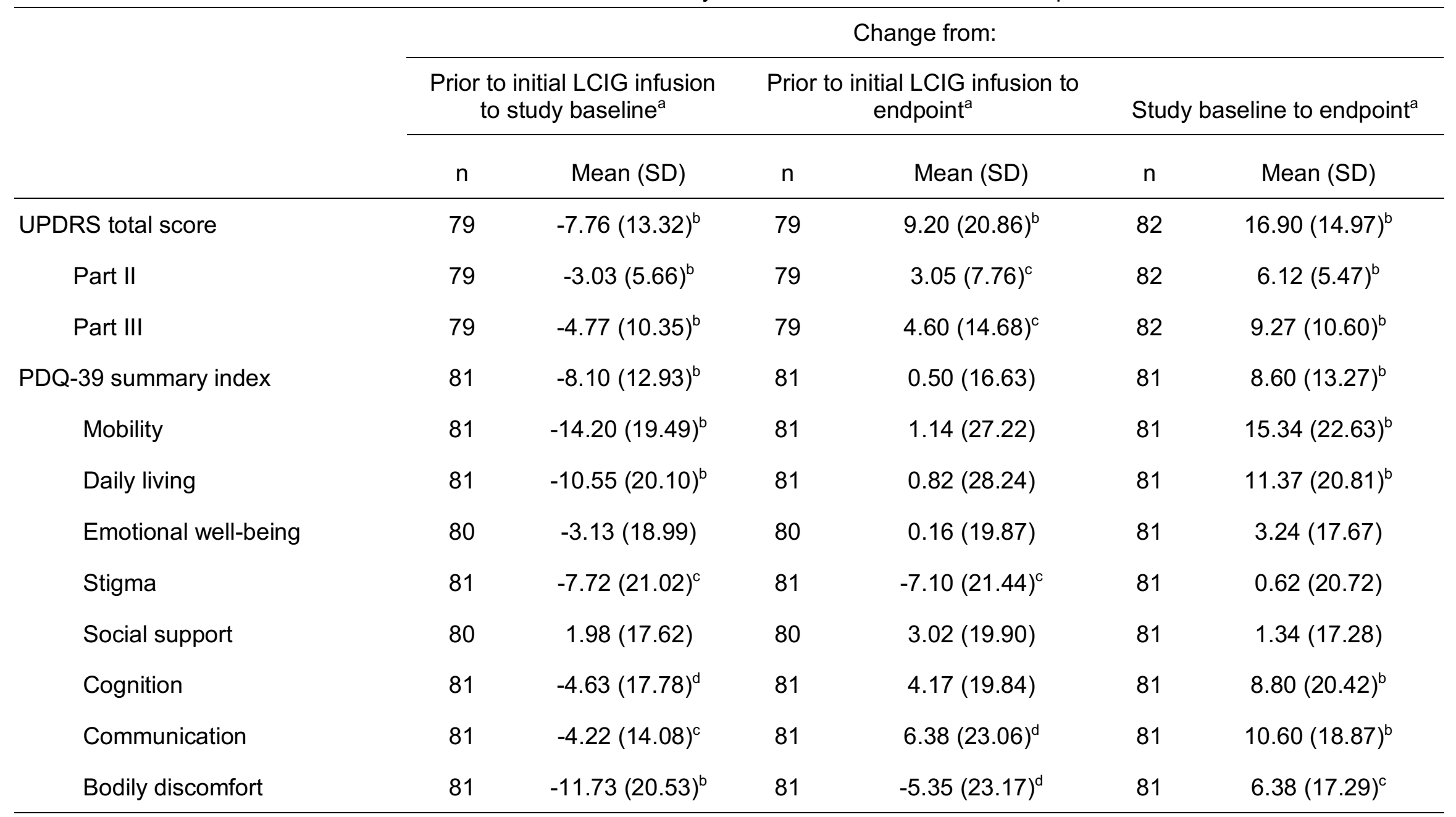


a"Prior to Initial LCIG Infusion" occurred in a previous study; "Study Baseline" refers to the last assessment in a previous study, which served as a baseline for this open-label extension study; "Endpoint" refers to measurements at the cutoff date for this open-label, extension study.

$$
{ }^{\mathrm{b}} P<0.001 \text {. }
$$

${ }^{\mathrm{c}} P<0.01$.

${ }^{\mathrm{d}} P<0.05$. 
SUPPLEMENTAL FIG. 1. Percent of patients with $\geq 1$ device complaint each year.

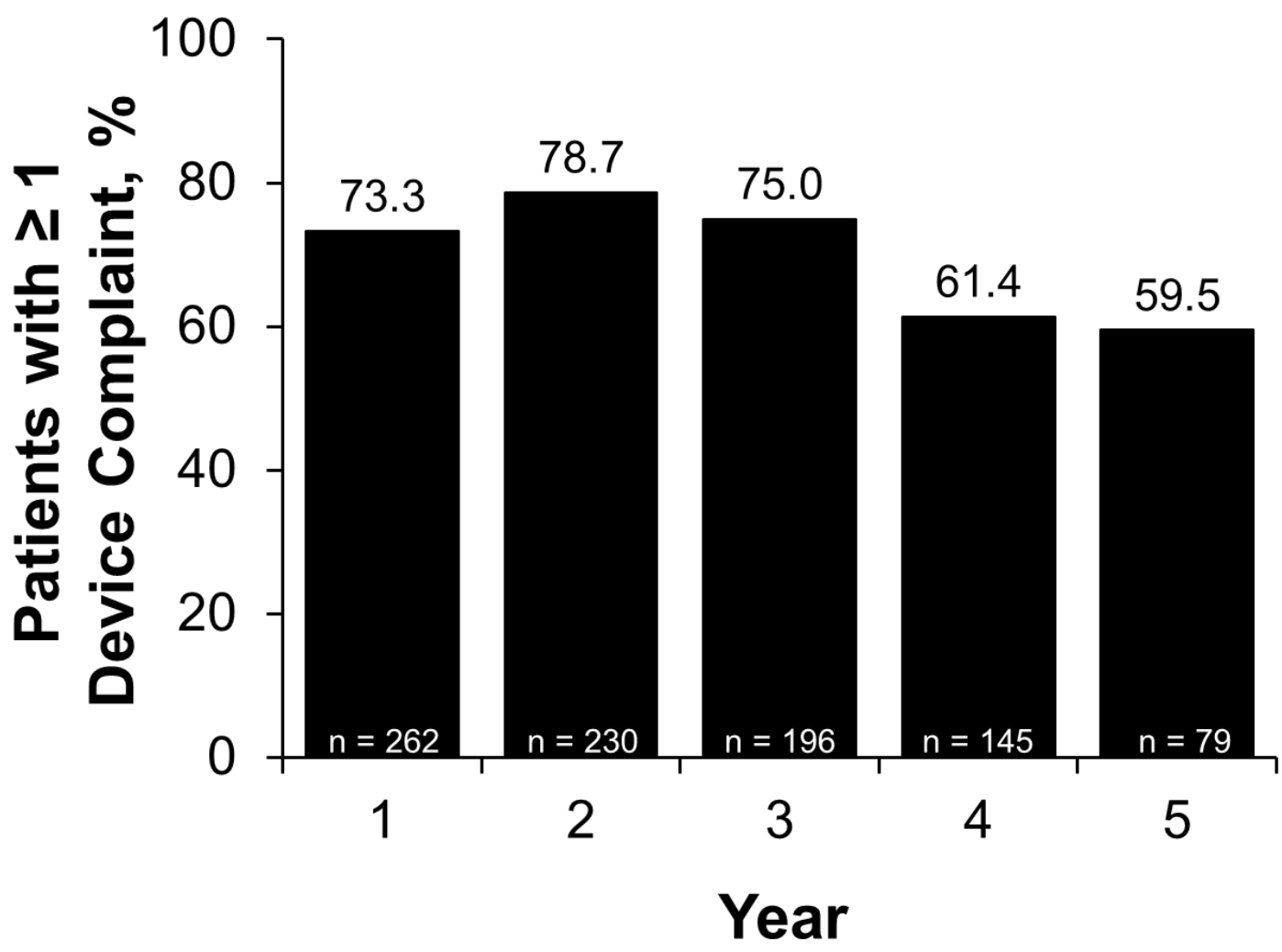


SUPPLEMENTAL FIG. 2. Patients receiving either PEG or $\mathrm{J}$ tube replacements based on (a) the total number of tube replacements during the study and (b) the number of tube replacements each year of the study. J, jejunal; PEG, percutaneous endoscopic gastrostomy.

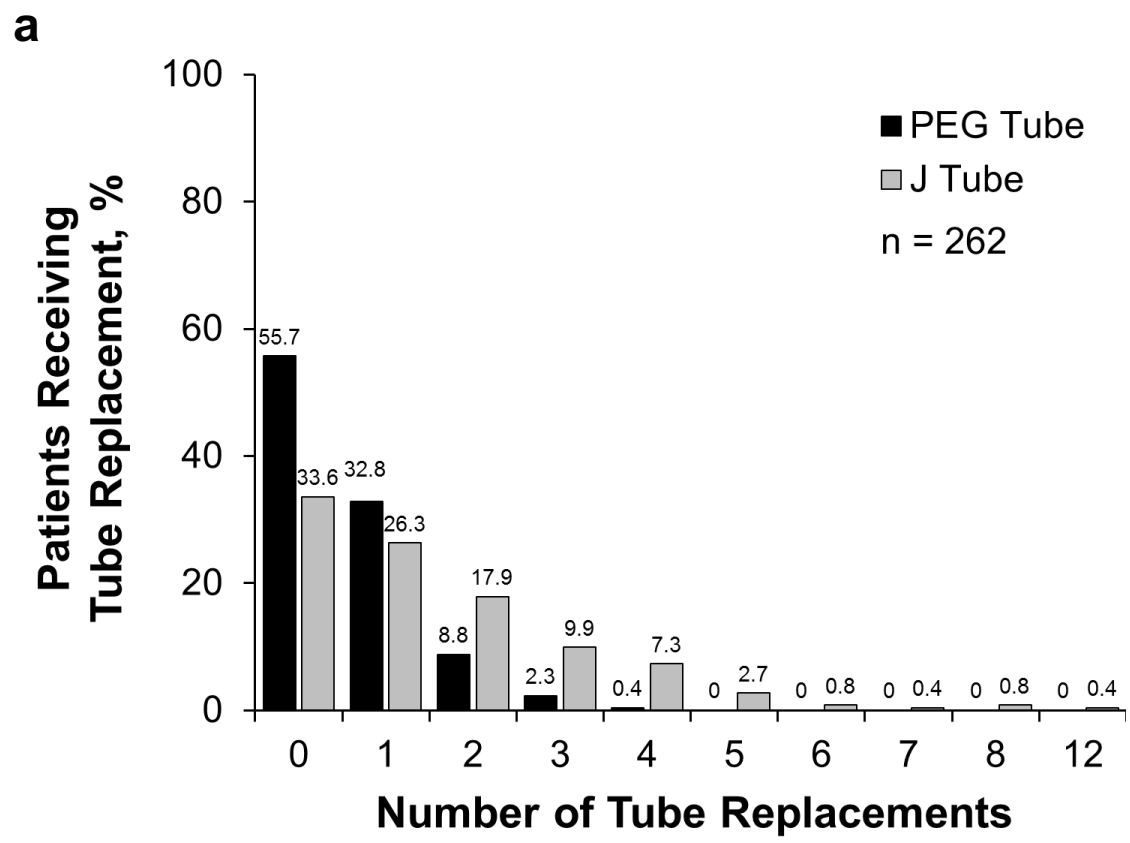

b

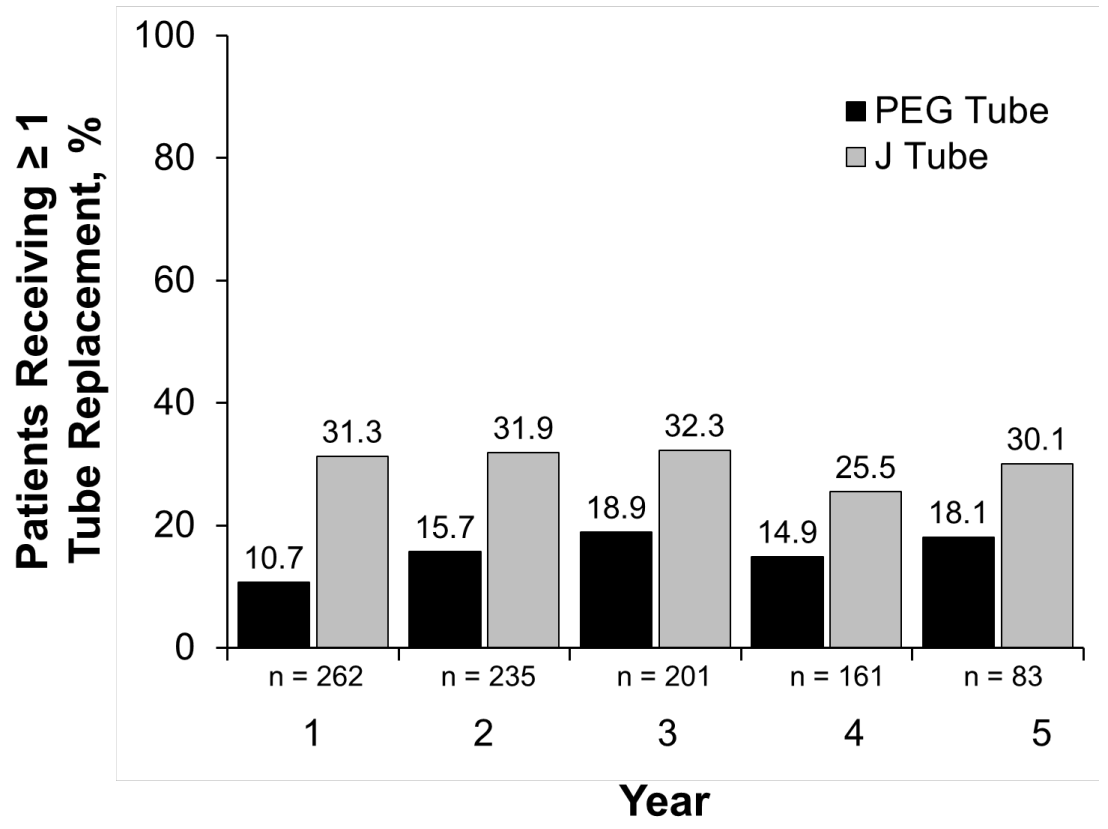

